Supporting Information

Synchronous Interface Modification and Bulk Passivation via One-step Cesium Bromide Diffusion Process for Highly Efficient Perovskite Solar Cells 


\section{Synchronous Interface Modification and Bulk Passivation via One-step Cesium Bromide Diffusion Process for Highly Efficient Perovskite Solar Cells}

Shangzheng Pang, Chunfu Zhang*, Hang Dong, Zeyang Zhang, Dazheng Chen, Weidong Zhu, Jingjing Chang, Zhenhua Lin, Jincheng Zhang, and Yue Hao

Wide Bandgap Semiconductor Technology Disciplines State Key Laboratory, Shaanxi Joint Key Laboratory of Graphene, School of Microelectronics, Xidian University, Xi'an 710071, China

Corresponding author: cfzhang@xidian.edu.cn 
Materials. All reagents were purchased from Sigma-Aldrich and used as received, including isopropanol (99.99\%), N,N-dimethylformamide (DMF) (99.99\%), 4-tertbutylpyridine (99.9\%), chlorobenzene (99.9\%), and acetonitrile (99.9\%). MAI (99.9\%, Dyesol), FAI (99.9\%, Dyesol), lead (II) iodide ( $\mathrm{PbI}_{2}, 99.999 \%$, Alfa), lead (II) chloride $\left(\mathrm{PbCl}_{2}, 99.999 \%\right.$, Alfa), cesium bromide $(\mathrm{CsBr}, 99.9 \%$,Alfa $)$ and Tin oxide $\left(\mathrm{SnO}_{2}, 15 \%\right.$ in $\mathrm{H}_{2} \mathrm{O}$ colloidal dispersion liquid, Alfa). Spiro-OMeTAD (Xi'an Polymer Light Technology Corp) and all the materials were used as received without further purification. The purity of the silver used for the top contact electrode is $99.99 \%$.

Device Characterization. The morphology measurement of the perovskite layers was taken by scanning electron microscopy (SEM) (JSM-7800F). X-ray diffraction (XRD) test was conducted on Bruker D8 Advance XRD. The transmittance and absorption spectra of different samples were recorded with an UV-visible spectrophotometer (Perkin-Elmer Lambda 950). Photoluminescence spectra were collected on an Edinburgh Instruments FLS920 Spectro fluorometer, and the excitation wavelength was $633 \mathrm{~nm}$. Photovoltaic performances were measured by using a Keithley 2400 source meter under simulated sunlight from XES-70S1 solar simulator matching the AM $1.5 \mathrm{G}$ standard with an intensity of $100 \mathrm{~mW} / \mathrm{cm}^{2}$. The system was calibrated against a NREL certified silicon reference solar cell. Incident photo-to-current conversion efficiencies (IPCEs) of PSCs were measured by the solar cell quantum efficiency measurement system (SCS10-X150, Zolix instrument. Co. Ltd). Steady photoluminescence was measured using the Pico Quant Fluotime 300 by using a 510 $\mathrm{nm}$ picosecond pulsed laser. XPS experiments were carried out on the Escalab 250Xi using monochromatic. Al-Ka $(1486.6 \mathrm{eV})$ as the radiation source. UPS experiments were carried out at the Escalab 250Xi using $\mathrm{He} \mathrm{I}(21.2 \mathrm{eV})$ as the excitation source. Photoluminescence spectra were collected on an Edinburgh Instruments FLS920 spectrofluorometer, and the excitation wavelength was $633 \mathrm{~nm}$. 


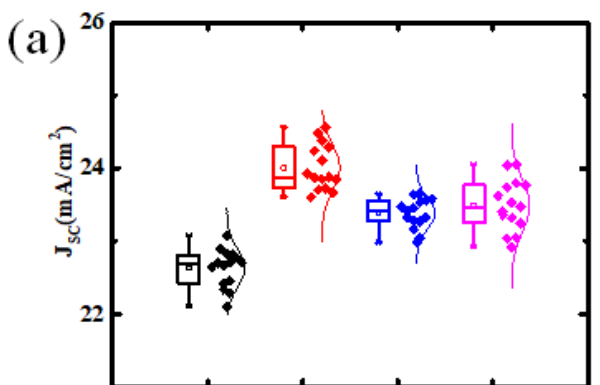

$\mathrm{SnO}_{2} \mathrm{SnO}_{2}: \mathrm{CsBr} \mathrm{SnO}_{2}: \mathrm{CsI} \mathrm{SnO}_{2}: \mathrm{CsCl}$

(c)

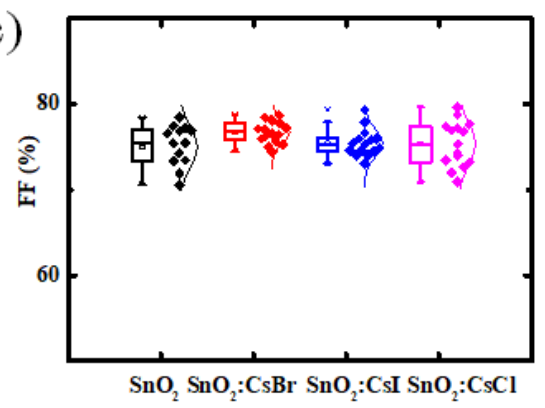

(b)

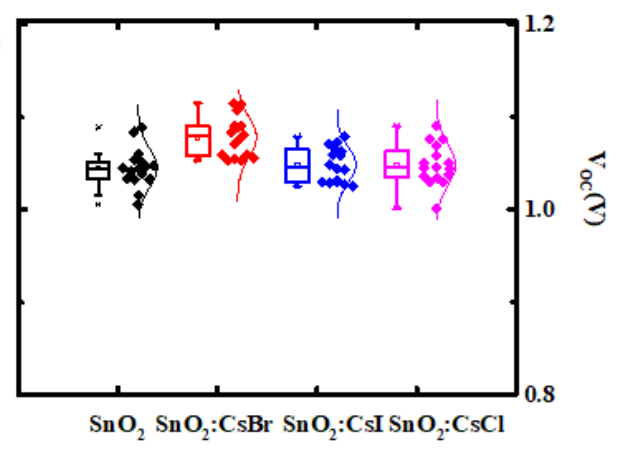

(d)

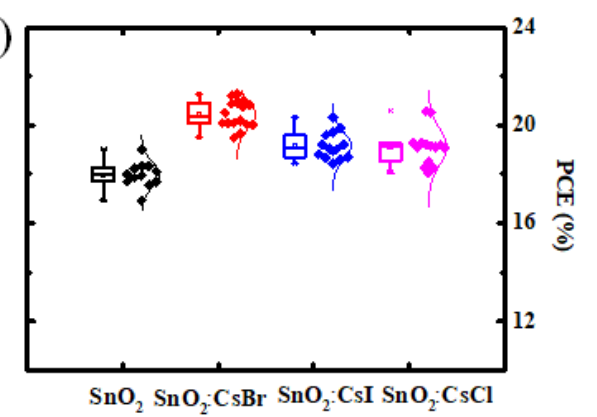

Figure S1. The statistics of PSCs electrical parameters with and without $\mathrm{CsCl}, \mathrm{CsBr}$, CsI modification. (a) short-circuit current $\left(J_{\mathrm{SC}}\right)$, (b) Open circuit voltage $\left(V_{\mathrm{OC}}\right)$, (c) Fill factor (FF) and (d) power conversion efficiency (PCE).

(a)

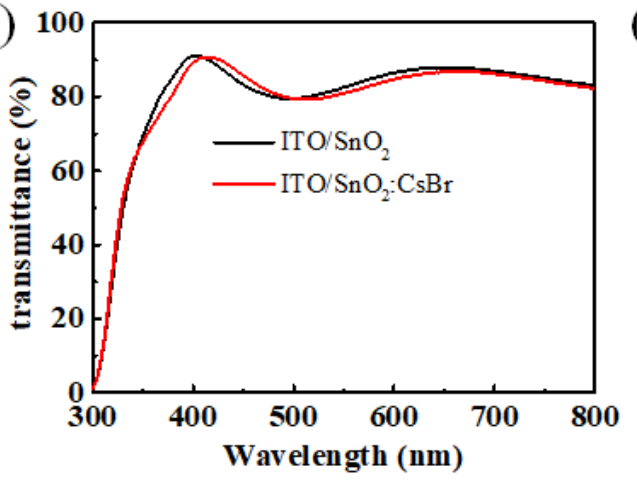

(b)

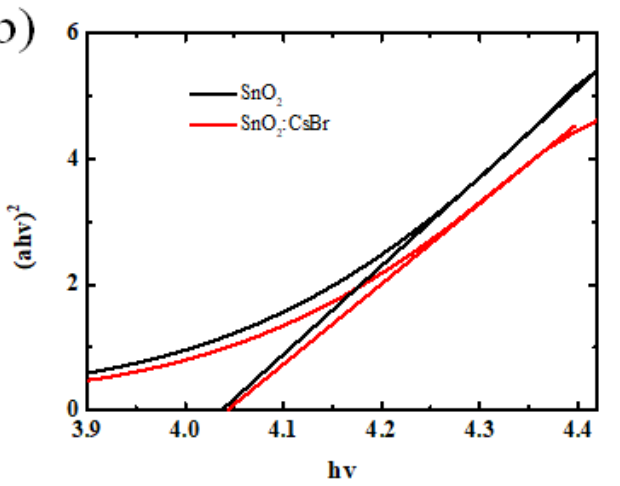

Figure S2. (a) The light transmittance and (b) optical bandgap of $\mathrm{SnO}_{2}$ and $\mathrm{SnO}_{2}: \mathrm{CsBr}$ samples 
(a)

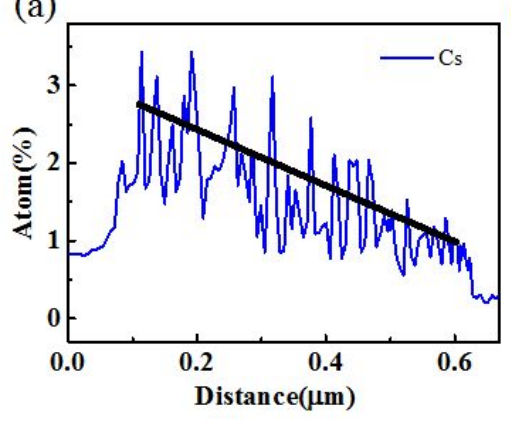

(b)

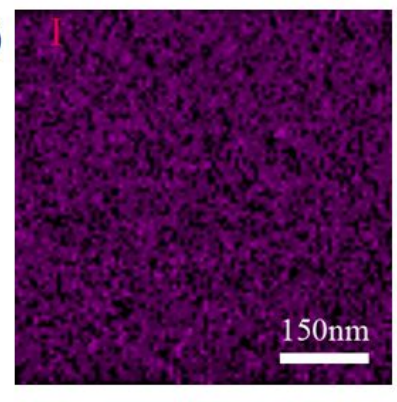

(c)

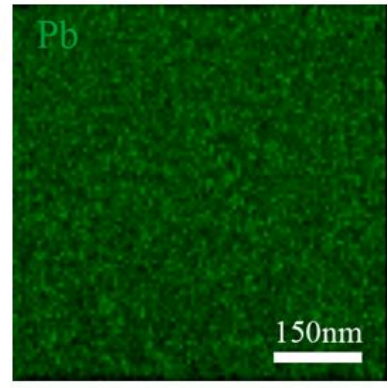

Figure S3. (a) Cs element distribution percent along the diffusion distance in the devices, the $0.1 \mu \mathrm{m}$ position is the interface of $\mathrm{CsBr}$ and perovskite layer (b-c) Cross-sectional EDS mapping images of perovskite film on $\mathrm{CsBr}$ substrate.

(a)

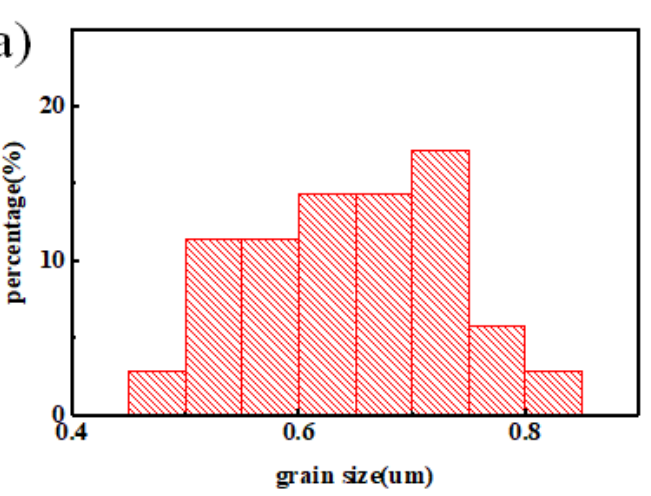

(b)

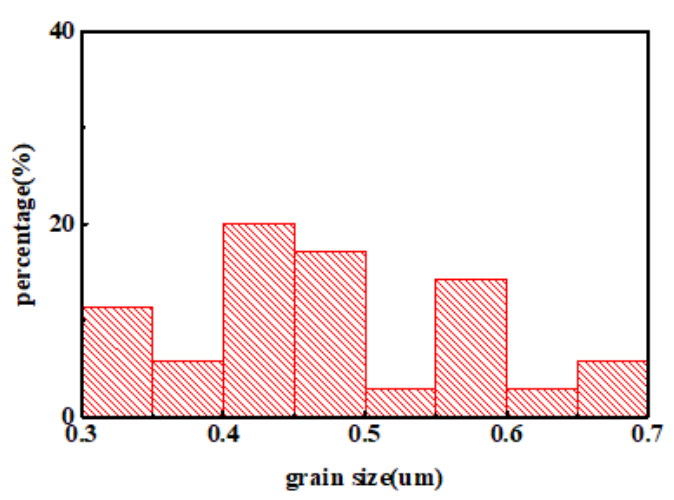

Figure S4. The grain size statistics of perovskite film based on (a) $\mathrm{SnO}_{2}: \mathrm{CsBr}$ and (b) $\mathrm{SnO}_{2}$ substrates

(a)

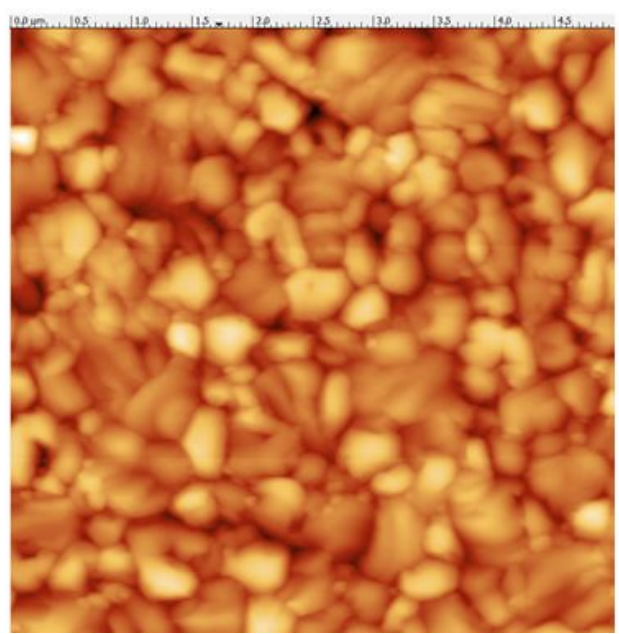

(b)

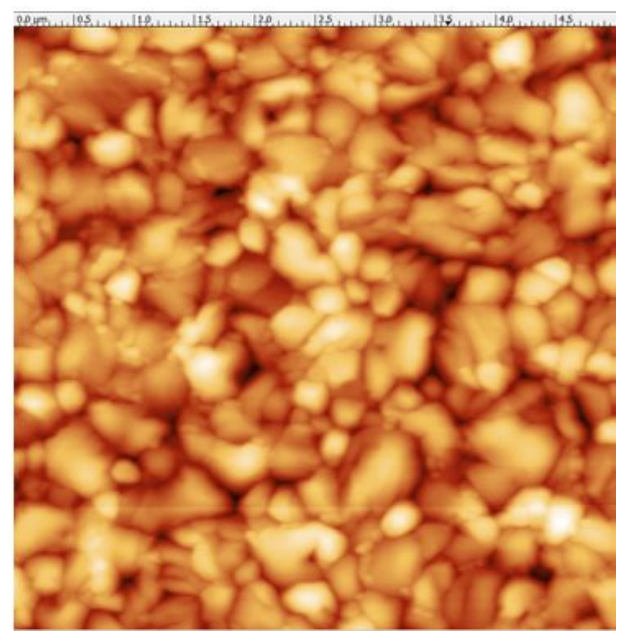

Figure S5. (a) AFM image of perovskite on $\mathrm{SnO}_{2}$ surface (b) $\mathrm{SnO}_{2}: \mathrm{CsBr}$ surface 


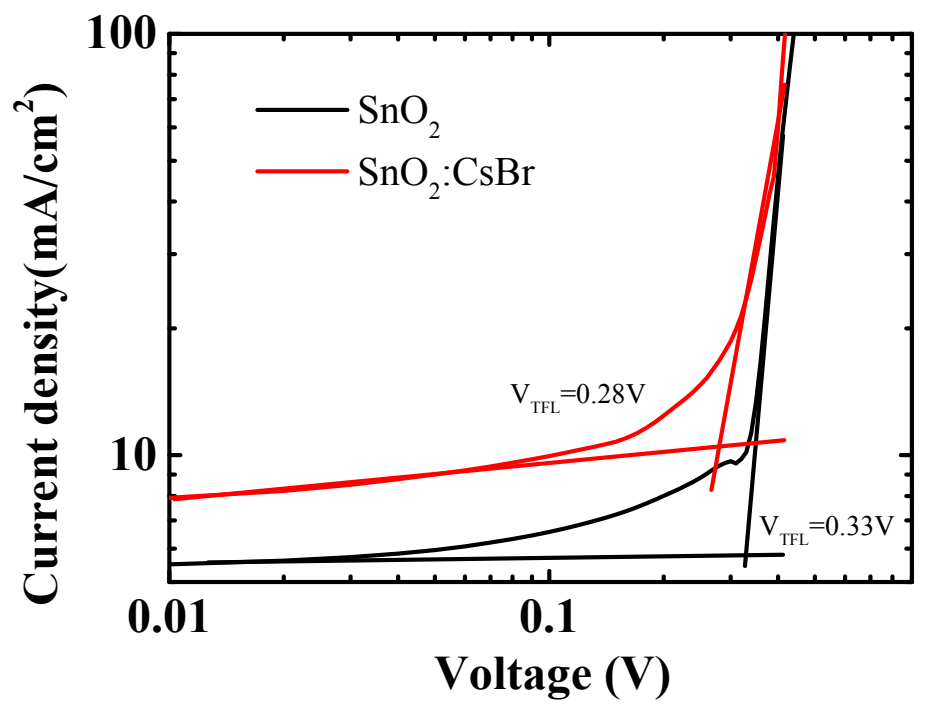

Figure S6. $J-V$ plots for the perovskite film on $\mathrm{SnO}_{2}: \mathrm{CsBr}$ and $\mathrm{SnO}_{2}$ layer using the space-charge-limited current model under illumination with device structure of ITO/ ETLs/Perovskite/PCBM/Ag

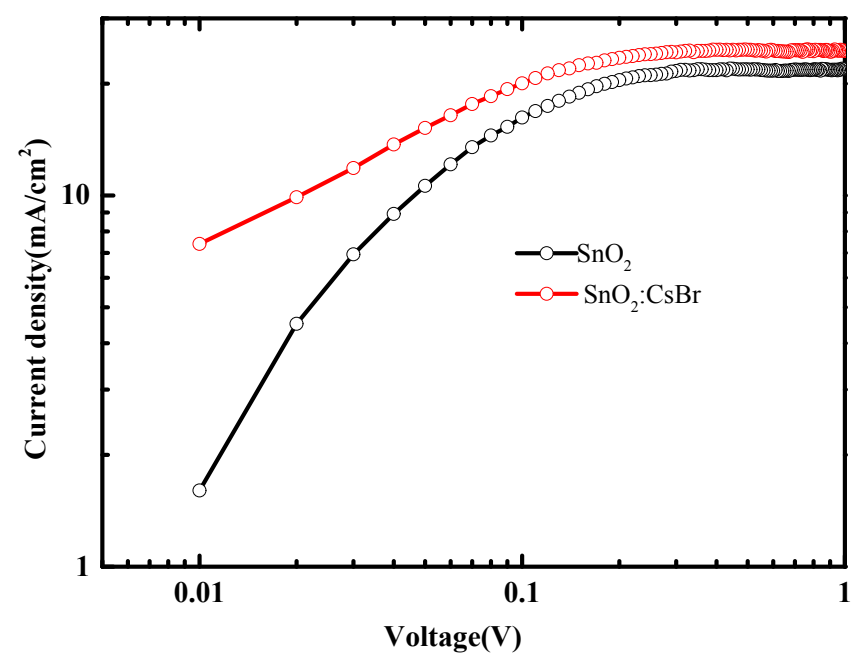

Figure $\mathrm{S} 7 . J_{\mathrm{ph}}-V_{\text {eff }}$ curves of $\mathrm{SnO}_{2}: \mathrm{CsBr}$ and $\mathrm{SnO}_{2}$ based devices 

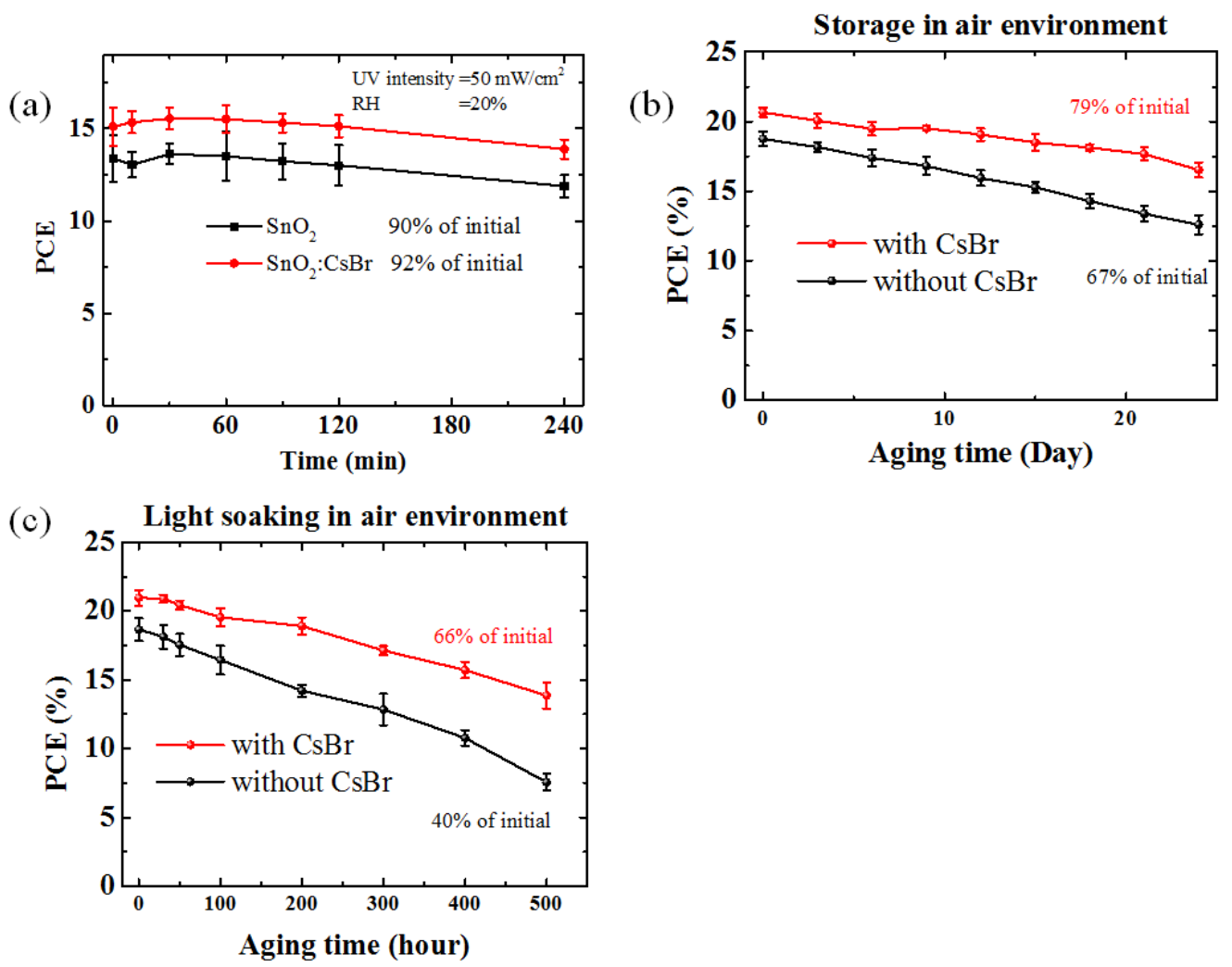

Figure S8. (a) UV-light stability (b) the shelf-life stability and (c) light stability (full sun) in air environment of unencapsulated devices with and without $\mathrm{CsBr}$

(a)

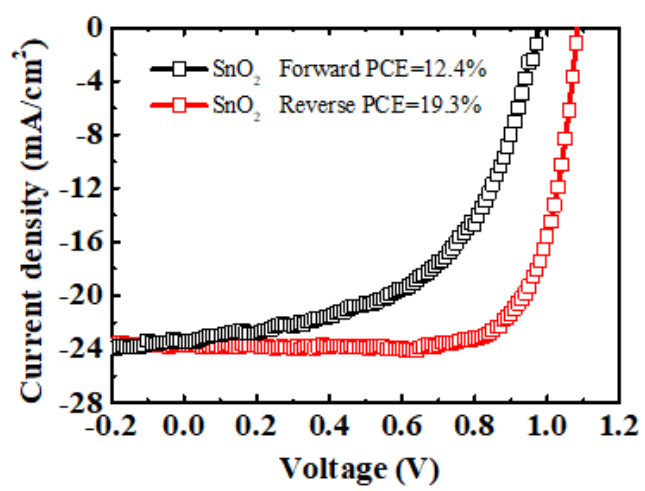

(b)

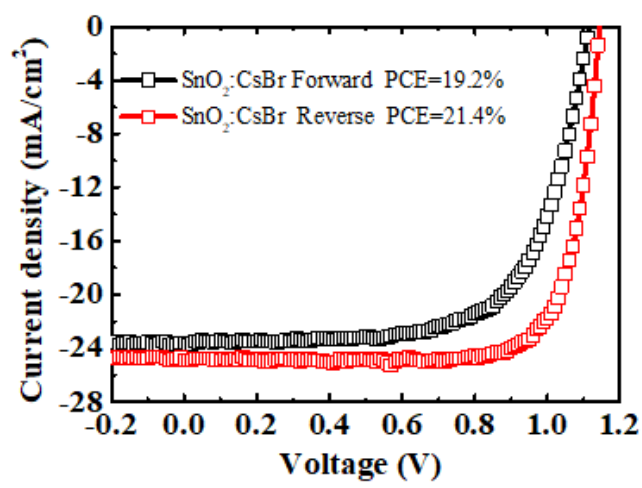

Figure S9. the Hysteresis data of perovskite solar cells (a) without and (b) with $\mathrm{CsBr}$ 